\title{
Brain Structures and Activity During a Working Memory Task Associated with Internet Addiction Tendency in Young Adults
}

Saeid Sadeghi ( $\square$ saeidsadeghi.psychologist@gmail.com )

Shahid Beheshti University

Hikaru Takeuchi

Tohoku University

Bita Shalani

Tarbiat Modares University

Yasuyuki Taki

Tohoku University

Rui Nouchi

Tohoku University

Ryoichi Yokoyama

Tohoku University

Yuka Kotozaki

Tohoku University

Seishu Nakagawa

Tohoku University

Atsushi Sekiguchi

Tohoku University

Kunio lizuka

Tohoku University

Sugiko Hanawa

Tohoku University

Tsuyoshi Araki

Tohoku University

Carlos Makoto Miyauchi

Tohoku University

Kohei Sakaki

Tohoku University

Takayuki Nozawa

Tohoku University

Shigeyuki lkeda 
Tohoku University

\section{Susumu Yokota}

Tohoku University

\section{Magistro Daniele}

Tohoku University

\section{Yuko Sassa}

Tohoku University

Ryuta Kawashima

Tohoku University

\section{Research Article}

Keywords: Internet addiction Tendency, Brain structures, Brain activity, Working memory task

Posted Date: February 4th, 2021

DOI: https://doi.org/10.21203/rs.3.rs-153035/v1

License: (c) (i) This work is licensed under a Creative Commons Attribution 4.0 International License. Read Full License 


\section{Abstract}

An increasing number of young people use internet excessively over the last decades, which leads to adverse impacts on individuals and society. The structural and functional brain characteristics associated with the excessive use of the internet have attracted substantial research attention in the past decade; however, due to the small sample sizes of past studies, many findings are inconsistent. Also, the relationship between internet addiction tendency (IAT) and regional brain activity during working memory (WM), a critical cognitive function governing learned behavior, has not been explored.

In current study, we used voxel-based morphometry (VBM) and multiple regression analysis to assess the relationship between IAT score and regional gray and white matter volumes (rGMVs and rWMVs) and brain activity during a WM task in a large sample of healthy young adults $(n=1,154$, mean age, $20.71 \pm$ 1.78 years).

We found a significant positive correlation between IAT score and GMV of right supramarginal gyrus (rSMG) and significant negative correlations with WMVs of right temporal lobe (sub-gyral and superior temporal gyrus), right sublobar area (extra-nuclear and lentiform nucleus), right cerebellar anterior lobe, cerebellar tonsil, right frontal lobe (inferior frontal gyrus and sub-gyral areas), and the pons. Also, IAT was significantly and positively correlated with brain activity in the default-mode network (DMN), medial frontal gyrus, medial part of the superior frontal gyrus, and anterior cingulate cortex) during a 2-back WM task. Moreover, whole-brain analyses of rGMV showed significant effects of interaction between sex and the IAT scores in the area spreading around the left anterior insula and left lentiform. This interaction was moderated by positive correlation in females.

These results indicate that IAT is associated with (a) increased GMV in rSMG, which is involved in phonological processing, (b) decreased WMVs in areas of frontal, sublobar, and temporal lobes, which are involved in response inhibition, and (c) reduced task-induced deactivation of the DMN, indicative of altered attentional allocation.

\section{Introduction}

The internet is a necessity in many lives ${ }^{1,2}$. More than half of the world's population are internet users ${ }^{3}$, ${ }^{4}$. Excessive internet use is associated with negative psychological consequences such as poor life satisfaction ${ }^{5,6}$, anxiety and aggression ${ }^{7,8}$, low self-esteem and depression ${ }^{9,10}$, and alcohol abuse ${ }^{11,12}$. Physical health problems such as sleep problems ${ }^{13-15}$ and social functioning impairments such as poor academic performance ${ }^{16,17}$ are other negative consequences of the excessive use of the internet.

Excessive internet use has also been associated with impaired executive functions ${ }^{18-22}$. Some studies have also indicated that internet users show working memory (WM) deficits compare to individuals without such behaviors ${ }^{20-23}$. 
WM is a central component of executive functioning ${ }^{24}$. It has been suggested that WM along with inhibition and shifting contribute to self-regulation ${ }^{25}$. Prior research suggests that WM is a significant predictor of ability to response inhibition ${ }^{26}$. WM deficits have been observed in individuals with hyperactivity and attention disorder (ADHD) and impulsivity 27,28 , substance-dependent individuals, including cocaine- ${ }^{29}$, alcohol- ${ }^{30}$, methamphetamine- ${ }^{31}$ and opioid-dependent individuals ${ }^{32}$. WM load interferes with individuals' ability to filter out irrelevant distractors ${ }^{33}$. Also, there are evidences of a significant conjunction between WM and response inhibition in the left inferior frontal gyrus ${ }^{34}$.

During cognitive tasks performance, the default-mode network (DMN) deactivated ${ }^{35,36}$. DMN is a set of brain regions (posterior cingulate/precuneus, medial prefrontal cortex) which considered a backbone of cortical integration ${ }^{37-40}$. Previous studies have revealed that task-induced deactivations occur within regions of the $\mathrm{DMN}$ during cognitive WM tasks ${ }^{41-45}$. In addition, a reduced magnitude of task-induced deactivation in the DMN is a characteristic of subjects with lower WM capacity and cognitive disinhibition 46,47 . Global Workspace Theory ${ }^{48}$ has helped researchers understand how WM relates to the DMN. In brief, the theory postulates that the central executive (CE) component of the WM model presides over cognitive slave systems to orchestrate conscious cognitive control of distracting stimuli. The $\mathrm{CE}$ is related to the executive control network and functions antagonistically to the DMN.

However, the relationship between characteristics of brain activity during a WM task and the tendency of people to IA has not been studied yet. One of the aims of this study is to understand the characteristics of brain activity during a WM task associated with IAT.

Research has tended to focus on internet addiction disorder (IAD) in pathological groups rather than IAT in healthy people groups. MRI studies have revealed that internet addiction (IA) scores negatively correlate with gray matter volumes (GMVs) in the anterior cingulate cortex (ACC), bilateral dorsolateral prefrontal cortex (DLPFC), orbitofrontal cortex (OFC), right middle frontal gyrus, supplementary motor area (SMA), cerebellum, left rostral ACC (rACC), and post-central gyrus (postCG) ${ }^{49-51}$. Lin, Zhou 52 also used diffusion tensor imaging (DTI) to investigate white matter integrity in adolescents with IAD. This study reported that people with higher IAD scores appeared to have lower white matter integrity in the fronto-temporal pathway connected through the external capsule. Takeuchi, Taki ${ }^{53}$ have shown that video game time is associated with increased mean diffusivity (MD) in the orbital frontal cortex and subcortical areas (putamen, pallidum, left hippocampus, caudate, right insula, and thalamus). Takeuchi, Taki ${ }^{54}$ also demonstrated in a longitudinal study that excessive internet use is associated with decreased verbal intelligence and a smaller developmental increase in regional gray and white matter volumes (rGMVs and rWMVs, respectively) across widespread brain areas in children.

Moreover, $\mathrm{fMRI}$ studies have shown that the most cortical dysfunctions in IAD are reported to be localized to the superior temporal gyrus ${ }^{55}$, cingulate cortex ${ }^{56}$, cerebellum ${ }^{57}$, and inferior frontal gyrus. In subcortical regions, functional alterations were often found in the brainstem and caudate ${ }^{58}$. Previous task-related fMRI studies on IAD have demonstrated differences in behavioral performance and 
differences in brain activation during cognitive tasks such as cue-reactivity paradigms in which subjects are exposed to internet or videogame stimuli to elicit a craving, probabilistic guessing paradigms in which subjects bet using cards or colors to analyze neural reward system dynamics in response to losses or wins, and cognitive control paradigms such as the GO-NOGO test for assessment of impulsivity and inhibitory control ${ }^{58}$.

Although such combined behavioral and neuroimaging studies have shown that IAD is associated with altered brain structure ${ }^{58}$, but due to small sample sizes ${ }^{59}$ and diversity in empirical research methods and paradigms in neuroimaging studies ${ }^{60}$ results are inconsistent and often are not replicated. Also, previous studies have all focused on the group of people with IAD, and the study of IAT in healthy people has been neglected. This study with a large sample size and focusing on the tendency of IA in healthy people can increase our knowledge about the nature of the phenomenon of IA. For these reasons, future studies are warranted.

The purpose of this study was thus to investigate these issues by assessing the effects of IAT on brain structure and activity during the n-back working memory task in a large sample of healthy young adults. Knowledge of the brain structure and function abnormalities and association between these abnormalities and IAT is helpful to identify possible interventions and pharmacotherapies to treat IA.

On the basis of the previous studies, we hypothesized that higher IAT scores may be associated with structural abnormalities in the frontal and temporal lobe and subcortical areas known to contribute to addiction vulnerability ${ }^{52,61-63}$. We also hypothesized that lower task-induced deactivation (TID) in the DMN during WM may be associated with a higher IAT score. This hypothesis is based on previous findings that suggest that TID in the DMN is associated with altered brain glutamatergic excitability and GABA inhibition ${ }^{64}$, that the glutamatergic neurons play a critical role in the reward system ${ }^{65}$, and that glutamatergic and GABAergic abnormalities are primary neurobiological characteristics in individuals with addiction ${ }^{66-68}$. We also hypothesized that lower TID in the DMN during WM may be associated with a higher IAT score, which is supported by previous studies that showed reduced task-induced deactivation in the DMN during working memory tasks in psychiatric patients ${ }^{69-71}$.

\section{Materials And Methods}

\section{Participants}

A total of 1,154 healthy right-handed young adults ( 666 males, mean age 20.79 years, SD $=1.89$ years and 488 females, mean age 20.60 years, SD = 1.61 years) participated in this study as part of our ongoing project to explore the associations among brain imaging characteristics, cognitive functions, aging, genetics, and daily habits. Indeed, from our database, we used the data from 1,154 subjects that had questionnaire data about internet dependence, fMRI imaging data, and behavioral data of the N-back task without apparent artifacts. Therefore, these 1,154 subjects are those who were included in the study after these exclusions were considered. 
All subjects were undergraduate or postgraduate students from Tohoku University in Japan. All had normal vision and none had a history of neurological or psychiatric illness. None reported recent use of any psychoactive drugs or other drugs that could negatively impact cognitive abilities. History of psychiatric illnesses and recent drug use were assessed by our laboratory's routine questionnaire. We used the Edinburgh Handedness Inventory to evaluate handedness in subjects ${ }^{72}$. The Ethics Committee of Tohoku University approved all procedures, which were performed in accordance with relevant guidelines and regulations. Written informed consent was obtained from each subject for the projects in which they participated. Descriptions in this subsection are adapted from a previous study using similar methods ${ }^{73}$.

\section{Internet Addiction Tendency assessment}

We used the Japanese version of Young's IAT scale to assess condition severity ${ }^{74}$. This IAT instrument consists of 20 items answered on a 1-5 scale from $1=$ "rarely" to $5=$ "always". The scale is selfadministered and requires 5 to 10 minutes. The IAT scale minimum and maximum scores are 20 and 100, with higher scores reflecting a greater tendency toward internet addiction. The Japanese version of this scale has demonstrated high reliability and validity 75 .

\section{fMRI task}

Functional MRI was used to map brain activity during working memory. The n-back task is a widely used task consisting of 0-back (simple cognitive processing) and 2-back (working memory) conditions. In the 2-back task, subjects viewed a series of stimuli presented sequentially (one of four Japanese vowels) and were instructed to judge if a target stimulus appearing "2" presentations earlier was the same as the current stimulus by pushing a button. In the 0-back task, subjects were instructed to determine whether a presented letter was the same as the target stimulus by pushing a button (Figure 1). We used a simple block design. Descriptions in this subsection were mostly adapted from our previous studies using similar methods ${ }^{73,76}$.

In this study, our focus was TID in the DMN. TID in the DMN occurs in mostly similar areas regardless of whether the task is 2-back or 0-back, although there are differing magnitudes. Furthermore, differences in brain activity between patients with schizophrenia and control subjects were similar regardless of whether the task was a 0-back task or 2-back. These included areas of DMN (i.e., subtracting the activity during the 0-back task from the brain activity during the 2-back task substantially eliminates group differences) ${ }^{77,78}$. Therefore, we did not analyze the contrast of 2-back - 0-back, as was done in another study that focused on TID in the DMN ${ }^{78}$.

\section{Image acquisition}

The MRI acquisition methods were described in our previous study ${ }^{79}$. Briefly, all MRI data were acquired using a 3T Philips Achieva scanner. Diffusion-weighted data were acquired using a spin-echo EPI 
sequence $\left(\mathrm{TR}=10293 \mathrm{~ms}, \mathrm{TE}=55 \mathrm{~ms}, \mathrm{FOV}=22.4 \mathrm{~cm}, 2 \times 2 \times 2 \mathrm{~mm}^{3}\right.$ voxels, 60 slices, SENSE reduction factor $=2$, number of acquisitions $=1$ ). The diffusion weighting was isotropically distributed along 32 directions $\left(b\right.$ value $\left.=1,000 \mathrm{~s} / \mathrm{mm}^{2}\right)$. In addition, three images with no diffusion weighting ( $b$ value $=0$ $\mathrm{s} / \mathrm{mm}^{2}$ or $\mathrm{b}=0$ images) were acquired using a spin-echo EPI sequence (TR $=10293 \mathrm{~ms}, \mathrm{TE}=55 \mathrm{~ms}, \mathrm{FOV}$ $=22.4 \mathrm{~cm}, 2^{\prime} 2^{\prime} 2 \mathrm{~mm}^{3}$ voxels, 60 slices). For the n-back session, 174 functional volumes were obtained 73. For more details, see our previous study ${ }^{53}$.

\section{Preprocessing of structural data}

Preprocessing of the structural and functional data was performed using Statistical Parametric Mapping software (SPM12; Wellcome Department of Cognitive Neurology, London, UK) implemented in MATLAB (Mathworks, Inc., Natick, MA). For analyses, T1-weighted structural images of each individual were segmented using the new segmentation algorithm implemented in SPM12 and normalized to Montreal Neurological Institute (MNI) space to yield images with $1.5 \times 1.5 \times 1.5 \mathrm{~mm}^{3}$ voxels using the diffeomorphic anatomical registration through exponentiated lie algebra registration process implemented in SPM12. In addition, we performed a volume change correction (modulation) ${ }^{80}$. Subsequently, generated rGMV and rWMV images were smoothed by convolution using an isotropic Gaussian kernel of $8 \mathrm{~mm}$ full width at half maximum. These descriptions were mostly adapted from our

previous study using similar methods. For full descriptions of these procedures, see our previous work ${ }^{73}$.

\section{Pre-processing and data analysis for functional activation data}

Pre-processing and data analysis of functional activation data were performed using SPM. The following procedures for functional activation data analysis were reproduced from our previous study, as described previously ${ }^{81}$. Prior to analysis, BOLD images were re-aligned and resliced to the mean BOLD image of the run. They were then corrected for slice timing, co-registered, and resliced to the voxel space of images of diffusion tensor imaging. All images were subsequently normalized using a previously validated ${ }^{82}$ twostep segmentation algorithm of the diffusion images and the previously validated diffeomorphic anatomical registration through exponentiated lie algebra-based registration process. The voxel size of normalized BOLD images was $3^{\prime} 3^{\prime} 3 \mathrm{~mm}^{3}$ and taken to the second-level analyses of functional activities. The full description of these procedures are provided in our previous study ${ }^{76}$.

A design matrix was fitted to each participant with one regressor for each task condition (0-, 2-back in the n-back task) using the standard hemodynamic response function. The design matrix weighted each raw image according to its overall variability, to reduce the impact of movement artifacts ${ }^{83}$. The design matrix was fit to the data for each participant individually. After estimation, beta images were smoothed ( $8 \mathrm{~mm}$ full-width half-maximum) and taken to the second level or subjected to a random effect analysis. We removed low-frequency fluctuations using a high-pass filter and a cutoff value of $128 \mathrm{~s}$. The individual-level statistical analyses were performed using a general linear model. 
In the individual analyses, we focused on activation related to the condition (0-back or 2-back versus rest). The resulting maps representing brain activity during the working memory condition (2-back) and simple cognitive processing condition (0-back) for each participant were then forwarded for group analysis.

The fMRI images with artifacts based on the visual inspection had been removed from the images. Thorough instruction to prevent motion during the scan was given to educated participants. Other exclusions based on motion parameters were not performed in this study.

In a previous study, we validated normalization procedures of fMRI using diffusion tensor images using SPM $8{ }^{84}$. Our internal preliminary survey also showed these procedures work better using SPM8. Conversely, VBM procedures work better with SPM12. In other words, the segmentation of the diffusion images obtained, which were part of our preprocessing procedures of $\mathrm{fMRI}$, were not adequate for SPM12. Misclassifications that were apparent by visual inspection were systematically found when SPM 12 was used. In the second-level analysis, the use of SPM8 or SPM12 does not affect the results of TFCE based on permutation.

Generally, thorough instructions and thorough fixation by the pad were provided to prevent head motion during the scan as much as possible, and we utilized the software to reduce the impact of movements ${ }^{83}$, as described in the subsection below.

Thus, we did not exclude any subject from the fMRI analyses based on excessive motion that did not cause evident artifacts during the scan. The subjects were young adults and the scan duration was very brief. Only the maximum movement of several subjects detracted from the original point, and in one of the directions exceeded $3 \mathrm{~mm}$. Removing these subjects from analyses did not substantially alter the significant results of the present study.

Similarly, the subjects enrolled in the study were educated young adults, and thorough instruction and sufficient practice was provided. Subjects whose responses were properly recorded showed acceptable accuracies and only seven subjects showed accuracies lower than $80 \%$ in the 0-back or 2-back task (but accuracies were at least $50 \%$ or greater). Removing these subjects also did not substantially alter the significant results of the present study.

\section{Effects of interaction between sex and the score of Young's IAT scale on imaging measures}

We also performed a supplementary investigation of the potential regions displaying significant effects of interaction between the subject's sex and score on the Young's IAT scale (that is, we investigated whether some regions showed sex-related differences in the correlations patterns based on the Young's IAT scale score). For this purpose, we performed whole-brain analyses of covariance (ANCOVAs). The dependent variables in these analyses were same as those in the whole-brain multiple regression analyses that were conducted to investigate the correlation with score of Young's IAT scale in each voxel across sexes. In these whole-brain ANCOVAs, sex was a group factor (using the full factorial option in 
SPM8), whereas and all other covariates are same as those of the abovementioned whole-brain multiple regression analyses. In addition, all covariates were modeled to enable unique relationships with imaging measures (dependent variables) (using the interactions option in SPM8) for each sex. The interaction between sex and the score of Young's IAT scale (contrasts of [the score of the Young IAT scale for males, the effect of the score of the Young IAT scale for females] were [-1 1] or [1 -1]) were assessed using tcontrasts. Correction for multiple comparisons was performed using the same method used in the wholebrain multiple regression analyses.

\section{Supplemental methods}

Supplemental analyses of the comparison between subjects using Young's IAT scale.

In accordance with the considerable literature available that classified subjects based on the Young's IAT scale score, we also divided subjects into two groups (IAT score $\geq 50$ and IAT score $<50$ ). This classification was used to compare dependent variables between those who used the internet excessively and those who used it less frequently. We hypothesized that excessive use of the internet would be associated with additional changes in brain structure and functional characteristics. For this reason, we also conducted the supplemental analyses of comparisons between subjects who scored $\geq 50$ using Young's IAT scale and those who scored $<50$ using Young's IAT scale, on the basis of the criteria described previously ${ }^{74}$.

For this comparison, we conducted multiple regression analyses in which all dependent and independent variables of the main analyses remained the same, except that Young's IAT score was replaced by the dichotomized value (Young's IAT scale $\geq 50=1$, Young's IAT scale $<0$ ).

Supplemental region of interest (ROI) analyses of the associations between activity in key nodes of the $D M N$ and IAT scores.

We conducted a supplemental partial correlation analyses of the associations between mean beta estimates of functional ROls of important nodes of the DMN and IAT after controlling for covariates. In these analyses, ROI masks were defined by the areas that are mostly significantly deactivated during the 2-back task using an appropriate threshold that successfully segregated each area in the representative DMN nodes for the 63 subjects from which the template of normalization was created (when there were multiple clusters in one area, those that showed the strongest statistical values at the peak were selected). The mean beta estimates of the 2-back task as well as the 0-back task within each ROI were extracted. For these analyses, control variables were same as those of the covariates in the whole-brain multiple regression analyses in the main text.

ROls were mPFC (peak coordinate: $x=-6, y=57, z=-6$, T score threshold $=15,425$ voxels), PCC/precuneus (peak coordinate: $x=-6, y=-57, z=12$, T score threshold $=15,305$ voxels), left hippocampus (peak coordinate: $x=-27, y=-21, z=-24, T$ score threshold $=9,27$ voxels), right hippocampus (peak coordinate: $x=24, y=-15, z=-27$, T score threshold $=9,66$ voxels), left 
temporoparietal junction (peak coordinate: $x=-45, y=-72, z=21$, T score threshold $=7,322$ voxels), and right temporoparietal junction (peak coordinate: $x=54, y=-69, z=27$, T score threshold $=7,32$ voxels).

Results with a threshold having $\mathrm{p}<0.05$, and corrected for the false discovery rate (FDR) using the twostage sharpened method ${ }^{85}$, were considered statistically significant.

\section{Statistical analysis}

Statistical analyses of imaging data were performed with SPM8. Structural whole-brain multiple regression analyses were performed to investigate associations of IAT scores with rGMV and rWMV. Age, sex, and total intracranial volume calculated using voxel-based morphometry (for details of calculation see ${ }^{86}$ ) were added as covariates.

For the functional images, we used multiple regression analysis to investigate the relationship between IAT score and brain activity levels during the 0-back, 2-back, and 2-back-0-back tasks. Age, sex, n-back task accuracy, and n-back task reaction time were entered into the multiple regression model as covariates.

A multiple comparison correction was performed using threshold-free cluster enhancement (TFCE) ${ }^{87}$ with randomized (5,000 permutations) nonparametric testing using the TFCE toolbox (http://dbm.neuro.uni-jena.de/tfce/). We applied a threshold of family-wise error corrected at $\mathrm{P}<.05$. SPM8 was used for analyses because of better compatibility with TFCE software and our in-house scripts 54.

\section{Results}

\section{Behavioral results}

There was no significant difference in mean age between sexes, but independent sample t-tests revealed a significant difference in the IAT score. Moreover, there was no significant difference in 2-back accuracy and reaction time between sexes. The distribution of IAT scores by sex is presented in Figure 2, and the ttest results are presented in Table 1.

Furthermore, to investigate the relationship between IA and 2-back accuracy and 2-back reaction time, Pearson's correlations were conducted. There were no significant correlations between IA and 2-back accuracy $(r=0.029, p=0.33)$ and 2-back reaction time $(r=-0.042, p=0.15)$.

\section{Structural results}

Voxel-based morphometry (VBM) revealed a significantly correlation between IAT score and rGMV of right supramarginal gyrus ( $\mathrm{rSMG}$ ) among the entire cohort (Table 2 and Figure 3 ) as well as significant negative correlations between IAT score and rWMVs of right temporal lobe (sub-gyral and superior temporal gyrus), right sublobar region (extra-nuclear and lentiform nucleus), right cerebellum anterior 
lobe, cerebellar tonsil, right frontal lobe (inferior frontal gyrus and sub-gyral), and pons (Table 3 and Figure 4).

\section{fMRI results}

Multiple regression analysis revealed that IAT scores were significantly and positively correlated with brain activity during the 2-back task in the medial frontal gyrus, superior frontal gyrus, and medial part of the ACC (Table 4 and Figure 5). This cluster of significant correlation mostly belonged to areas that were deactivated during the 2-back task (Table 4).

\section{Effects of interaction between sex and IAT score}

There were only significant effects in the interaction between sex and the score of Young's IAT scale in the whole-brain analyses of rGMV. The significant effects of interaction were found in the area around the left anterior insula and left lentiform nucleus $(p=0.021$, corrected, $x, y, z=-24,16.5,9$, TFCE score $1402.35,3791 \mathrm{~mm}^{3}$ under the threshold of $p<0.05$, corrected) (Figure 6).

This interaction had a positive correlation in females $(r=0.1822, p<.001)$ and no correlations in males $(r=-0.054, p=0.163)$.

\section{Supplemental results}

\section{Supplemental analyses of the comparison between subjects using Young's IAT scale}

The rGMV analysis revealed there were no significant correlations between rGMV and the dichotomized value (Young's IAT scale $\geq 50=1$, Young's IAT scale $<0$ ). However, this analysis revealed that subjects who scored $\geq 50$ using Young's IAT scale had a tendency of greater rGMV in a similar area of significant correlation between rGMV and Young's IAT scale (right IPL, $x, y, z=42,-49.5,51,1166 \mathrm{~mm}^{3}$ under the threshold of $p<0.001$, uncorrected).

The rWMV analysis revealed that subjects who scored $\geq 50$ using Young's IAT scale had lower rWMV, with significant negative correlations between rWMV and the dichotomized value (Young's IAT scale $\geq 50=1$, Young's IAT scale $<0)$, in the left frontal white matter area $(p=0.026$, corrected, $x, y, z=-21,46.5,6$, $\left.7395 \mathrm{~mm}^{3}\right)$, in the right frontal white matter area $(p=0.036$, corrected, $x, y, z=31.5,25.5,-1.5$, $\left.2333 \mathrm{~mm}^{3}\right)$, and in the white matter area in the cerebellum and the brain stem $(p=0.040$, corrected, $x, y$, $z=19.5,-24,-30,2749 \mathrm{~mm}^{3}$ ). These significant areas are mostly included and overlapping with areas that had significant negative correlations between continuous scoring values for Young's IAT scale and rWMV. A corrected $p$-value threshold of $p<0.05$ was used for all analyses.

The $\mathrm{fMRI}$ analysis revealed that there were no significant correlations. However, subjects who scored $\geq 50$ using Young's IAT scale showed a tendency of greater brain activity during the 2-back task in a similar area of significant correlation between brain activity during the 2-back task and Young's IAT scale (mPFC, $x, y, z=3,51,-6,972 \mathrm{~mm}^{3}$ under the threshold of $p<0.001$, uncorrected). 
These results suggest similar but weaker tendencies of the correlations of the dichotomized value (Young's IAT scale $\geq 50=1$, Young's IAT scale $<0$ ) as compared with the significant correlations between the continuous score of Young's IAT scale and neuroimaging measures.

Supplemental ROI analyses of the associations between activity in key nodes of the DMN and IAT scores

After correcting for multiple comparisons, the partial correlation analyses showed that the IAT score significantly and positively correlated with brain activity (2-back) of the ROI of the MPFC, left hippocampus, and right hippocampus. Similar tendencies were observed for the brain activity (2-back) of $\mathrm{ROI}$ of the PCC/precuneus, and for brain activity (0-back) in the ROI of the left and right hippocampus (Supplemental Table 1).

\section{Discussion}

To the best of our knowledge, this is the first study to investigate the associations between internet addiction tendency and brain activity during a working memory task in healthy young adults. First, VBM showed a positive association of IAT score with GMV across the supramarginal gyrus and negative associations of IAT score with rWMVs in the right inferior frontal gyrus (rIFG) and sub-gyral frontal lobe, extra-nuclear, lentiform nucleus, right cerebellum anterior lobe, cerebellar tonsil, sub-gyral temporal lobe, superior temporal gyrus, and pons. These rWMV correlations with IAT score are consistent with our original hypothesis that IAT is strongly associated with abnormal brain structures in fronto-striatal areas 61,62 . However, cortical areas outside the frontal lobe were significant.

In this study, the association between IAT scores and brain activity during the WM task was observed only in the anterior part of the DMN (the MPFC and contingent regions), but not in the posterior DMN. We added a supplemental ROI analyses that investigated the brain activity of functional ROIs of important nodes based on the DMN and IAT scores (Supplemental Methods, Supplemental Results, and Supplemental Table 1). This analysis showed there was a significant positive correlation between brain activity defined by the IAT score and brain activity observed in the MPFC and bilateral hippocampus. In addition, brain activity of the PCC/precuneus during the 2-back task, also showed a marginally insignificant positive correlation. Thus, it is difficult to conclude that there is no correlation between the IAT score and brain activity of the posterior DMN. Whether this relatively weaker result for the posterior DMN is due to statistical fluctuation or other reasons remains unclear. However, previous studies have demonstrated that reduced TID in the DMN during working memory tasks in the elderly or psychiatric patients is seen in both the posterior and anterior parts of the DMN (Whitfield-Gabrieli et al., 2009; Sambataro et al., 2010). Moreover, our supplemental analysis revealed the tendency of positive correlation between the mean brain activity of the cluster of significant correlation between the IAT score and brain activity in the MPFC in this study. We also showed the accuracy of the 2-back task after controlling for age, sex, and framewise displacement during the scan (partial correlation coefficient: $-0.049, p=0.096)$. However, reaction time during the 2 -back task did not show such tendencies. These 
findings suggest that the TID in the anterior part of the DMN is also associated with cognitive processes during working memory.

In the present study, the GMV of right supramarginal gyrus (rSMG) was significantly positive correlated with IAT score, consistent with recent studies implicating the supramarginal gyrus in addiction ${ }^{36,88}$. Also in accord with functions in addiction, this region is responsible for phonological processing ${ }^{89}$ and our recent study revealed that frequent internet use in children is associated with a decrease in verbal intelligence ${ }^{54}$. In most previous studies, however, there was a negative correlation between GMV volume and addiction (35-39), while our current study found positive correlations between IAT and GMV in the left caudate. These discrepancies among studies may be due to differences in sample groups. Our sample group included only university students, who are of above average intelligence and would use the internet more frequently for learning and education. Another possible explanation is greater internet accessibility in recent years (via smart phones, Wi-Fi, etc.). The possible mechanisms are diverse. For example, the development of the smartphone and accessibility Wi-Fi have made it easy to use the internet under the condition of dual tasks (e.g., engaging in the internet while walking), which might lead to changes in functions and structures of attention-related areas in the users. Faster internet speed might allow faster access to the verbal and visual information, which may in turn lead to structural changes in relevant brain areas in users. However, these explanations are speculative and require future study.

Our study also revealed negative correlations between the IAT score and rWMVs in frontal, temporal and sublobar areas, regions responsible for response inhibition, visuospatial/visuomotor functions, and reward system functions. These findings are consistent with our hypothesis that IAT would correlate with WMVs in fronto-striatal areas. As stated, fronto-striatal circuits are critical for the emergence of addictive behaviors. Previous studies have demonstrated contributions of the right inferior frontal gyrus (rIFG) to addiction ${ }^{90,91}$ likely through critical functions in response inhibition, decision making, target detection, and inhibitory control ${ }^{92}$. Impulsive responses are inhibited by engaging frontal-basal ganglia pathways involving the rIFG, striatum, pre-supplementary motor area (pre-SMA), and subthalamic nucleus (STN) ${ }^{93}$. The cortex is connected to the subthalamic nucleus via a hyperdirect pathway as well as by a slower indirect pathway in which cortical outputs are first sent to the striatum, then passed to the globus pallidus pars externa, and finally to the STN ${ }^{94}$. Previous studies have shown that both the hyperdirect and indirect basal ganglia pathways are critical for response inhibition ${ }^{93,95}$. Thus, negative correlations between IAT score and rWMVs of frontal, temporal, and sublobar areas may reflect poor response control for internet use.

There was also a negative correlation between IAT score and WMVs of right temporal lobe (sub-gyral and superior temporal gyrus). This result is consistent with previous findings indicating that addiction is associated with abnormalities in the cerebral cortex, including the temporal cortex. For instance, Fortier et al. ${ }^{96}$ showed that alcoholism in adults is itself linked to a decrease in cortical thickness in the temporal, frontal, and occipital cortical regions and these changes correlated positively with the severity of abuse. Further, significant negative correlations between rWMVs and IAT scores were found in the sublobar 
regions and lobes of the cerebellum. Moulton, Elman ${ }^{97}$ posited that the cerebellum, as an intermediary between motor function and reward, motivation, and cognitive control systems would have important roles in the etiology of addiction.

These negative correlations between regional WMVs and IAT scores may reflect reduced myelination or loss of WM integrity within these pathways. As detailed in our previous studies ${ }^{84,98}$, changes in myelination, glial cell number, glial cell size, and number of axon collaterals can all influence WMV. Therefore, decreased regional WMV may reflect reduced myelination, glial cell number/size, and (or) axonal number, which in turn impedes both regional neural transmission and neural transmission among networks. Thus, decreases in these physiological components in fronto-striatal pathways, right temporal lobe, sublobar regions, and cerebellum lobe, and ensuing transmission deficits may lead to impaired response inhibition and both visuospatial/visuomotor and reward system dysfunction. The present findings thus advance our understanding of WM dysfunction in IAT among young adults. More intense IAT is associated with WMV reductions in core brain regions responsible for response inhibition, visuospatial/visuomotor functions, and reward.

\section{Conclusions}

In conclusion, we demonstrate significant association of IAT severity with both white and gray matter volumes, as well as with DMN activity during a working memory task. Internet addiction tendency is characterized by increased gray matter volume in the rSMG brain region. This region is responsible for phonological processing, decreased rWMVs in brain regions involved in inhibition, visuospatial/visuomotor functions, and the reward system. Moreover, IAT is correlated with reduced TID of the DMN. Collectively, our findings suggest that IAT may share neural mechanisms with other types of addiction.

\section{Limitations and further research}

This study has several limitations. First, the cross-sectional design precludes establishment of causal relationships between IAT and changes in specific brain structures and activity patterns during a WM task. Second, as this study cohort consisted only of healthy young adults at a relatively high educational level, these findings may be extrapolated to the general population. Age, intellectual ability, education level, and general health can also strongly influence brain structures and increase sensitivity of the analyses ${ }^{99}$.

\section{References}

1. Cheng $\mathrm{C}$ and Li AY-I. Internet addiction prevalence and quality of (real) life: A meta-analysis of 31 nations across seven world regions. Cyberpsychology, Behavior, and Social Networking 2014; 17 : 755-760. 
2. unicef. Perils and possibilities: growing up online. New York: United Nations Children's Fund. https://www.unicef.org/endviolence/endviolenceonline/files/UNICEF_Growing-up-online.pdfS, 2016.

3. Zenebe $\mathrm{Y}$, Kunno $\mathrm{K}$, Mekonnen $\mathrm{M}$, et al. Prevalence and associated factors of internet addiction among undergraduate university students in Ethiopia: a community university-based cross-sectional study. BMC psychology 2021; 9: 1-10.

4. Miniwatts Marketing Group. World internet usage and population statistics. Internet World Stats 2018; https://www.internetworldstats.com/stats.htm.

5. Bozoglan B, Demirer V and Sahin I. Loneliness, self-esteem, and life satisfaction as predictors of Internet addiction: A cross-sectional study among Turkish university students. Scandinavian journal of psychology 2013; 54: 313-319.

6. Shahnaz I and Karim AR. The impact of Internet addiction on life satisfaction and life engagement in young adults. Universal Journal of Psychology 2014; 2: 273-284.

7. Capetillo-Ventura N and Juárez-Treviño M. Internet addiction in university medical students. Medicina universitaria 2015; 17: 88-93.

8. Lai C, Mak K, Watanabe $\mathrm{H}$, et al. The mediating role of Internet addiction in depression, social anxiety, and psychosocial well-being among adolescents in six Asian countries: a structural equation modelling approach. Public health 2015; 129: 1224-1236.

9. Bahrainian SA, Alizadeh KH, Raeisoon M, et al. Relationship of Internet addiction with self-esteem and depression in university students. Journal of preventive medicine and hygiene 2014; 55: 86.

10. Rabadi L, Ajlouni M, Masannat S, et al. The Relationship between Depression and Internet Addiction among University Students in Jordan. Journal of Addiction Research \& Therapy Rabadi et al, $J$ Addict Res Ther 2017; 8: 6.

11. Ko CH, Yen J-Y, Yen CF, et al. The association between Internet addiction and problematic alcohol use in adolescents: the problem behavior model. CyberPsychology \& Behavior 2008; 11: 571-576.

12. Morioka $\mathrm{H}$, Itani $\mathrm{O}$, Osaki $\mathrm{Y}$, et al. The association between alcohol use and problematic internet use: A large-scale nationwide cross-sectional study of adolescents in Japan. Journal of epidemiology 2017; 27: 107-111.

13. Younes $\mathrm{F}$, Halawi $\mathrm{G}$, Jabbour $\mathrm{H}$, et al. Internet addiction and relationships with insomnia, anxiety, depression, stress and self-esteem in university students: a cross-sectional designed study. PloS one 2016; 11: e0161126.

14. Bener A, Yildirim E, Torun P, et al. Internet Addiction, Fatigue, and Sleep Problems Among Adolescent Students: a Large-Scale Study. International Journal of Mental Health and Addiction 2018: 1-11.

15. Kuo SY, Chen YT, Chang YK, et al. Influence of internet addiction on executive function and learning attention in Taiwanese school-aged children. Perspectives in psychiatric care 2018.

16. Usman NH, Alavi M and Shafeq SM. Relationship between internet addiction and academic performance among foreign undergraduate students. Procedia-Social and Behavioral Sciences 2014; 114: 845-851. 
17. Kumar S, Kumar A, Badiyani B, et al. Relationship of internet addiction with depression and academic performance in Indian dental students. Clujul Medical 2018; 91: 300.

18. Park M-H, Park E-J, Choi J, et al. Preliminary study of Internet addiction and cognitive function in adolescents based on IQ tests. Psychiatry Res 2011; 190: 275-281.

19. Zhou Z-H, Yuan G-Z, Yao J-J, et al. An event-related potential investigation of deficient inhibitory control in individuals with pathological Internet use. Acta Neuropsychiatrica 2010; 22: 228-236.

20. Xiong $\mathrm{J}$ and Yao $\mathrm{L}$. The research of event-related potentials in working memory of the juvenile internet addiction. In: E-Health Networking, Digital Ecosystems and Technologies (EDT), 2010 International Conference on 2010, pp.93-95. IEEE.

21. Zhou Z, Zhu H, Li C, et al. Internet addictive individuals share impulsivity and executive dysfunction with alcohol-dependent patients. Frontiers in behavioral neuroscience 2014; 8: 288.

22. Nie J, Zhang W, Chen J, et al. Impaired inhibition and working memory in response to internet-related words among adolescents with internet addiction: A comparison with attention-deficit/hyperactivity disorder. Psychiatry research 2016; 236: 28-34.

23. Zhou Z, Zhou $\mathrm{H}$ and Zhu $\mathrm{H}$. Working memory, executive function and impulsivity in Internet-addictive disorders: A comparison with pathological gambling. Acta Neuropsychiatrica 2016; 28: 92-100.

24. Baddeley A. Working memory: Theories, models, and controversies. Annual review of psychology 2012; 63: 1-29.

25. Hofmann W, Schmeichel BJ and Baddeley AD. Executive functions and self-regulation. Trends in cognitive sciences 2012; 16: 174-180.

26. Traverso L, Mantini C, Usai MC, et al. The Relationship Between Inhibition and Working Memory In Preschoolers: Evidence For Different Inhibitory Abilities. In: EAPCogSci 2015.

27. Fosco WD, Kofler MJ, Groves NB, et al. Which 'Working'Components of Working Memory aren't Working in Youth with ADHD? Journal of Abnormal Child Psychology 2020: 1-14.

28. Kofler MJ, Singh LJ, Soto EF, et al. Working memory and short-term memory deficits in ADHD: A bifactor modeling approach. Neuropsychology 2020.

29. Berry J, Van Gorp WG, Herzberg DS, et al. Neuropsychological deficits in abstinent cocaine abusers: preliminary findings after two weeks of abstinence. Drug and alcohol dependence 1993; 32: 231-237.

30. Beatty WW, Katzung VM, Moreland VJ, et al. Neuropsychological performance of recently abstinent alcoholics and cocaine abusers. Drug and alcohol dependence 1995; 37: 247-253.

31. McKetin R and Mattick R. Attention and memory in illicit amphetamine users. Drug and alcohol dependence 1997; 48: 235-242.

32. Ersche KD, Clark L, London M, et al. Profile of executive and memory function associated with amphetamine and opiate dependence. Neuropsychopharmacology 2006; 31: 1036-1047.

33. Pratt N, Willoughby A and Swick D. Effects of working memory load on visual selective attention: behavioral and electrophysiological evidence. Frontiers in human neuroscience 2011; 5: 57. 
34. McNab F, Leroux G, Strand F, et al. Common and unique components of inhibition and working memory: an fMRI, within-subjects investigation. Neuropsychologia 2008; 46: 2668-2682.

35. Buckner RL, Andrews-Hanna JR and Schacter DL. The brain's default network: anatomy, function, and relevance to disease. 2008.

36. Ekhtiari H and Paulus M. Neuroscience for Addiction Medicine: From Prevention to RehabilitationMethods and Interventions. Elsevier, 2016.

37. Andrews-Hanna JR, Reidler JS, Sepulcre J, et al. Functional-anatomic fractionation of the brain's default network. Neuron 2010; 65: 550-562.

38. Kernbach JM, Yeo BT, Smallwood J, et al. Subspecialization within default mode nodes characterized in 10,000 UK Biobank participants. Proceedings of the National Academy of Sciences 2018; 115: 12295-12300.

39. Margulies DS, Ghosh SS, Goulas A, et al. Situating the default-mode network along a principal gradient of macroscale cortical organization. Proceedings of the National Academy of Sciences 2016; 113: 12574-12579.

40. Lopez-Persem A, Verhagen L, Amiez C, et al. The human ventromedial prefrontal cortex: sulcal morphology and its influence on functional organization. Journal of Neuroscience 2019; 39: 3627 3639.

41. Fox MD, Snyder AZ, Vincent JL, et al. The human brain is intrinsically organized into dynamic, anticorrelated functional networks. Proceedings of the National Academy of Sciences 2005; 102: 9673-9678.

42. Anticevic A, Cole MW, Murray JD, et al. The role of default network deactivation in cognition and disease. Trends in cognitive sciences 2012; 16: 584-592.

43. Bartova L, Meyer BM, Diers K, et al. Reduced default mode network suppression during a working memory task in remitted major depression. Journal of Psychiatric Research 2015; 64: 9-18.

44. Tomasi D, Ernst T, Caparelli EC, et al. Common deactivation patterns during working memory and visual attention tasks: An intra-subject fMRI study at 4 Tesla. Human brain mapping 2006; 27: 694705.

45. Mayer JS, Roebroeck A, Maurer K, et al. Specialization in the default mode: Task-induced brain deactivations dissociate between visual working memory and attention. Human brain mapping 2010; 31: 126-139.

46. Whitfield-Gabrieli S, Thermenos HW, Milanovic S, et al. Hyperactivity and hyperconnectivity of the default network in schizophrenia and in first-degree relatives of persons with schizophrenia. Proceedings of the National Academy of Sciences 2009; 106: 1279-1284.

47. Sambataro F, Murty VP, Callicott JH, et al. Age-related alterations in default mode network: impact on working memory performance. Neurobiology of aging 2010; 31: 839-852.

48. Baars BJ, Franklin S and Rams $\varnothing$ y TZ. Global workspace dynamics: cortical "binding and propagation" enables conscious contents. Frontiers in psychology 2013; 4: 200. 
49. Pan N, Yang Y, Du X, et al. Brain structures associated with internet addiction tendency in adolescent online game players. Frontiers in psychiatry 2018; 9: 67.

50. Yuan K, Qin W, Wang G, et al. Microstructure abnormalities in adolescents with internet addiction disorder. PloS one 2011; 6: e20708.

51. Wang $\mathrm{H}$, Jin $\mathrm{C}$, Yuan $\mathrm{K}$, et al. The alteration of gray matter volume and cognitive control in adolescents with internet gaming disorder. Frontiers in behavioral neuroscience 2015; 9: 64.

52. Lin F, Zhou Y, Du Y, et al. Abnormal white matter integrity in adolescents with internet addiction disorder: a tract-based spatial statistics study. PloS one 2012; 7: e30253.

53. Takeuchi H, Taki Y, Hashizume H, et al. Impact of videogame play on the brain's microstructural properties: Cross-sectional and longitudinal analyses. Mol Psychiatry 2016; 21: 1781-1789.

54. Takeuchi H, Taki Y, Asano K, et al. Impact of frequency of internet use on development of brain structures and verbal intelligence: Longitudinal analyses. Human brain mapping 2018; 39: 44714479.

55. Dong G, Lin X, Zhou H, et al. Cognitive flexibility in internet addicts: fMRI evidence from difficult-toeasy and easy-to-difficult switching situations. Addictive Behaviors 2014; 39: 677-683.

56. Dong G, Shen Y, Huang J, et al. Impaired error-monitoring function in people with internet addiction disorder: an event-related fMRI study. European addiction research 2013; 19: 269-275.

57. Jun L, Gao X-p, Isoken O, et al. Increased regional homogeneity in internet addiction disorder: a resting state functional magnetic resonance imaging study. Chinese medical journal 2010; 123 : 1904-1908.

58. Sepede G, Tavino M, Santacroce R, et al. Functional magnetic resonance imaging of internet addiction in young adults. World journal of radiology 2016; 8: 210.

59. Rogers JC and De Brito SA. Cortical and subcortical gray matter volume in youths with conduct problems: a meta-analysis. JAMA psychiatry 2016; 73: 64-72.

60. Kuss DJ and Griffiths MD. Internet and gaming addiction: a systematic literature review of neuroimaging studies. Brain sciences 2012; 2: 347-374.

61. Morein-Zamir S and Robbins TW. Fronto-striatal circuits in response-inhibition: Relevance to addiction. Brain research 2015; 1628: 117-129.

62. Brand M, Young KS and Laier C. Prefrontal control and Internet addiction: a theoretical model and review of neuropsychological and neuroimaging findings. Frontiers in human neuroscience 2014; 8: 375.

63. Hong S-B, Kim J-W, Choi E-J, et al. Reduced orbitofrontal cortical thickness in male adolescents with internet addiction. Behavioral and Brain Functions 2013; 9: 11.

64. Hu Y, Chen X, Gu H, et al. Resting-state glutamate and GABA concentrations predict task-induced deactivation in the default mode network. J Neurosci 2013; 33: 18566-18573.

65. Yoo JH, Zell V, Gutierrez-Reed N, et al. Ventral tegmental area glutamate neurons co-release GABA and promote positive reinforcement. Nature communications 2016; 7: 13697. 
66. Moeller SJ, London ED and Northoff G. Neuroimaging markers of glutamatergic and GABAergic systems in drug addiction: Relationships to resting-state functional connectivity. Neuroscience \& Biobehavioral Reviews 2016; 61: 35-52.

67. Herman BH, Frankenheim J, Litten RZ, et al. Glutamate and addiction. Springer, 2003.

68. Kalivas PW. Glutamate systems in cocaine addiction. Current opinion in pharmacology 2004; 4: 2329.

69. Sambataro F, Wolf ND, Giusti P, et al. Default mode network in depression: a pathway to impaired affective cognition? Clinical Neuropsychiatry 2013; 10.

70. Broyd SJ, Demanuele C, Debener S, et al. Default-mode brain dysfunction in mental disorders: a systematic review. Neuroscience \& biobehavioral reviews 2009; 33: 279-296.

71. Mohan A, Roberto AJ, Mohan A, et al. The Significance of the Default Mode Network (DMN) in Neurological and Neuropsychiatric Disorders: A Review. Yale J Biol Med 2016; 89: 49-57. 2016/08/10.

72. Oldfield RC. The assessment and analysis of handedness: the Edinburgh inventory. Neuropsychologia 1971; 9: 97-113.

73. Takeuchi $\mathrm{H}$, Taki $Y$, Hashizume $\mathrm{H}$, et al. Failing to deactivate: the association between brain activity during a working memory task and creativity. Neuroimage 2011; 55: 681-687.

74. Young KS. Caught in the net: How to recognize the signs of internet addiction-and a winning strategy for recovery. John Wiley \& Sons, 1998.

75. Osada H. Internet addiction in Japanese college students: Is Japanese version of Internet Addiction Test (JIAT) useful as a screening tool. Bulletin of Senshu University School of Human Sciences 2013; 3: 71-80.

76. Takeuchi H, Tomita H, Taki Y, et al. Cognitive and neural correlates of the 5-repeat allele of the dopamine D4 receptor gene in a population lacking the 7-repeat allele. Neuroimage 2015; 110: 124135.

77. Jansma J, Ramsey N, Van Der Wee N, et al. Working memory capacity in schizophrenia: a parametric fMRI study. Schizophr Res 2004; 68: 159-171.

78. Whitfield-Gabrieli S, Thermenos HW, Milanovic S, et al. Hyperactivity and hyperconnectivity of the default network in schizophrenia and in first-degree relatives of persons with schizophrenia. Proc Natl Acad Sci U S A 2009; 106: 1279-1284.

79. Takeuchi $\mathrm{H}$, Taki $\mathrm{Y}, \mathrm{Hashizume} \mathrm{H}$, et al. The association between resting functional connectivity and creativity. Cerebral Cortex 2012; 22: 2921-2929.

80. Ashburner J and Ridgway GR. Symmetric diffeomorphic modeling of longitudinal structural MRI. Frontiers in neuroscience 2013; 6: 197.

81. Takeuchi H, Tomita H, Taki Y, et al. A Common CACNA1C Gene Risk Variant has Sex-Dependent Effects on Behavioral Traits and Brain Functional Activity. Cereb Cortex 2019; 29: 3211-3219. DOI: 10.1093/cercor/bhy189. 
82. Takeuchi H, Taki Y, Thyreau B, et al. White matter structures associated with empathizing and systemizing in young adults. Neuroimage 2013; 77: 222-236.

83. Diedrichsen $\mathrm{J}$ and Shadmehr R. Detecting and adjusting for artifacts in fMRI time series data. Neuroimage 2005; 27: 624-634.

84. Takeuchi $\mathrm{H}$, Taki Y, Thyreau B, et al. White matter structures associated with empathizing and systemizing in young adults. Neuroimage 2013; 77: 222-236.

85. Benjamini Y, Krieger AM and Yekutieli D. Adaptive linear step-up procedures that control the false discovery rate. Biometrika 2006; 93: 491-507.

86. Takeuchi $\mathrm{H}$, Taki $\mathrm{Y}$, Hashizume $\mathrm{H}$, et al. The impact of television viewing on brain structures: crosssectional and longitudinal analyses. Cerebral Cortex 2015; 25: 1188-1197.

87. Smith SM and Nichols TE. Threshold-free cluster enhancement: addressing problems of smoothing, threshold dependence and localisation in cluster inference. Neuroimage 2009; 44: 83-98.

88. Fortier CB, Leritz EC, Salat DH, et al. Widespread effects of alcohol on white matter microstructure. Alcoholism: Clinical and Experimental Research 2014; 38: 2925-2933.

89. Petrides M. Neuroanatomy of language regions of the human brain. Academic Press, 2013.

90. Volkow ND, Fowler JS, Wang G-J, et al. Cognitive control of drug craving inhibits brain reward regions in cocaine abusers. Neuroimage 2010; 49: 2536-2543.

91. Wang X, Li B, Zhou X, et al. Changes in brain gray matter in abstinent heroin addicts. Drug and alcohol dependence 2012; 126: 304-308.

92. Schmidt A, Walter M and Borgwardt S. Impaired Cognition Control and Inferior Frontal Cortex Modulation in Heroin Addiction. Neuropathology of Drug Addictions and Substance Misuse. Elsevier, 2016, pp.1037-1047.

93. Li B, Friston KJ, Liu J, et al. Impaired frontal-basal ganglia connectivity in adolescents with internet addiction. Scientific reports 2014; 4: 5027.

94. Steiner $\mathrm{H}$ and Tseng KY. Handbook of basal ganglia structure and function. Academic Press, 2016.

95. Jahfari S, Waldorp L, van den Wildenberg WP, et al. Effective connectivity reveals important roles for both the hyperdirect (fronto-subthalamic) and the indirect (fronto-striatal-pallidal) fronto-basal ganglia pathways during response inhibition. Journal of Neuroscience 2011; 31: 6891-6899.

96. Fortier CB, Leritz EC, Salat DH, et al. Reduced cortical thickness in abstinent alcoholics and association with alcoholic behavior. Alcoholism: Clinical and Experimental Research 2011; 35: 21932201.

97. Moulton EA, Elman I, Becerra LR, et al. The cerebellum and addiction: insights gained from neuroimaging research. Addiction biology 2014; 19: 317-331.

98. Takeuchi H, Taki Y, Sassa Y, et al. Verbal working memory performance correlates with regional white matter structures in the frontoparietal regions. Neuropsychologia 2011; 49: 3466-3473.

99. Magistro D, Takeuchi H, Nejad KK, et al. The relationship between processing speed and regional white matter volume in healthy young people. PloS one 2015; 10: e0136386. 


\section{Tables}

Table 1. Comparison of IAT scores between males and females

\begin{tabular}{|c|c|c|c|c|c|c|c|c|}
\hline \multicolumn{2}{|l|}{ Variable } & Sex & $\underline{M}$ & $\underline{\text { SD }}$ & $\underline{\mathrm{MD}}$ & $d f$ & $t$ & $p$ \\
\hline \multirow{2}{*}{\multicolumn{2}{|c|}{ Age }} & Male & 20.79 & 1.89 & \multirow[t]{2}{*}{0.19} & \multirow[t]{2}{*}{1152} & \multirow[t]{2}{*}{1.87} & \multirow[t]{2}{*}{0.062} \\
\hline & & Female & 20.60 & 1.61 & & & & \\
\hline \multirow{2}{*}{\multicolumn{2}{|c|}{ Internet addiction tendency }} & Male & 41.32 & 13.10 & \multirow[t]{2}{*}{2.70} & \multirow[t]{2}{*}{1152} & \multirow[t]{2}{*}{3.52} & \multirow[t]{2}{*}{0.0001} \\
\hline & & Female & 38.62 & 12.58 & & & & \\
\hline \multirow{4}{*}{$\begin{array}{l}\text { Working } \\
\text { Memory }\end{array}$} & \multirow{2}{*}{$\begin{array}{l}\text { 2-back } \\
\text { accuracy }\end{array}$} & Male & 0.99 & 0.030 & \multirow[t]{2}{*}{-0.11} & \multirow[t]{2}{*}{1152} & \multirow[t]{2}{*}{-1.15} & \multirow[t]{2}{*}{0.25} \\
\hline & & Female & 1.10 & 2.36 & & & & \\
\hline & \multirow{2}{*}{$\begin{array}{l}2 \text {-back } \\
\text { reaction time }\end{array}$} & Male & 6688.07 & 1769.27 & \multirow{2}{*}{-4.78} & \multirow[t]{2}{*}{1152} & \multirow{2}{*}{$-\overline{0.387}$} & \multirow[t]{2}{*}{0.698} \\
\hline & & Female & 6729.85 & 1862.29 & & & & \\
\hline
\end{tabular}

Abbreviations: $\underline{\mathrm{M}}$, mean; $\underline{\mathrm{SD}}$, standard deviation; $\underline{\mathrm{MD}}$ mean differences; $\mathrm{df}$, degree of freedom

Table 2. Brain gray matter regions with a significant positive main effect of IAT score on volume

\begin{tabular}{|lllllll|}
\hline Anatomical area & \multicolumn{3}{l}{$\begin{array}{l}\text { MNI } \\
\text { coordinates }\end{array}$} & $\begin{array}{l}\text { TFCE } \\
\text { value }\end{array}$ & $\begin{array}{l}\text { Corrected } p \text { value } \\
\text { (FWE) }\end{array}$ & $\begin{array}{l}\text { Cluster size } \\
\left(\mathrm{mm}^{3}\right)\end{array}$ \\
\cline { 2 - 6 } & $\mathrm{X}$ & $\mathrm{Y}$ & $\mathrm{z}$ & & & 250 \\
\hline $\begin{array}{l}\text { Right supramarginal } \\
\text { gyrus }\end{array}$ & 63 & -23 & 47 & 1193.41 & 0.044 & 2 \\
\hline
\end{tabular}

Abbreviations: GM, gray matter: L, left: R, right; MNI, Montreal Neurological Institute; TFCE, threshold-free cluster enhancement

Table 3: Brain white matter regions with a significant negative main effect of IAT score on volume 


\begin{tabular}{|c|c|c|c|c|c|c|c|c|}
\hline \multirow[t]{2}{*}{ Cluster } & \multirow[t]{2}{*}{ Lobe (L/R) } & \multirow[t]{2}{*}{$\begin{array}{l}\text { Nearest WM } \\
\text { area }\end{array}$} & \multicolumn{3}{|c|}{$\begin{array}{l}\text { MNI } \\
\text { coordinates }\end{array}$} & \multirow[t]{2}{*}{$\begin{array}{l}\text { TFCE } \\
\text { value }\end{array}$} & \multirow{2}{*}{$\begin{array}{l}\text { Corrected } p \\
\text { value } \\
\text { (FWE) }\end{array}$} & \multirow{2}{*}{$\begin{array}{l}\text { Cluster } \\
\text { size } \\
\left(\mathrm{mm}^{3}\right)\end{array}$} \\
\hline & & & $x$ & $y$ & Z & & & \\
\hline \multirow[t]{3}{*}{1} & Temporal (R) & Sub-Gyral & 23 & -53 & 15 & 1742.07 & 0.007 & \multirow[t]{3}{*}{113825} \\
\hline & Sublobar (R) & Extra-Nuclear & 24 & -39 & 14 & 1635.53 & 0.008 & \\
\hline & Temporal (R) & $\begin{array}{l}\text { Superior } \\
\text { temporal gyrus }\end{array}$ & 42 & -35 & 6 & 1615.76 & 0.008 & \\
\hline \multirow[t]{3}{*}{2} & $\begin{array}{l}\text { Cerebellum } \\
\text { posterior (R) }\end{array}$ & $\begin{array}{l}\text { Cerebellar } \\
\text { tonsil }\end{array}$ & 14 & -47 & -44 & 1621.78 & 0.008 & \multirow[t]{3}{*}{42741} \\
\hline & Brain stem $(\mathrm{R})$ & Pons & 18 & -35 & -33 & 1618.07 & 0.008 & \\
\hline & $\begin{array}{l}\text { Cerebellum } \\
\text { anterior }(R)\end{array}$ & $\begin{array}{l}\text { cerebellum } \\
\text { anterior lobe }\end{array}$ & 21 & -44 & -39 & 1595.88 & 0.008 & \\
\hline \multirow[t]{3}{*}{3} & Frontal (R) & Sub-gyral & 27 & 21 & -11 & 1368.78 & 0.015 & \multirow[t]{3}{*}{10618} \\
\hline & Frontal (R) & Sub-gyral & 30 & 27 & 0 & 1321.91 & 0.017 & \\
\hline & Frontal (R) & $\begin{array}{l}\text { Inferior frontal } \\
\text { gyrus }\end{array}$ & 32 & 36 & -11 & 1176.26 & 0.026 & \\
\hline 4 & Sublobar (R) & $\begin{array}{l}\text { Lentiform } \\
\text { nucleus }\end{array}$ & 26 & 2 & -6 & 927.62 & 0.048 & 6.75 \\
\hline 5 & Sublobar (R) & $\begin{array}{l}\text { Lentiform } \\
\text { nucleus }\end{array}$ & 27 & 0 & -5 & 926.90 & 0.048 & 6.75 \\
\hline
\end{tabular}

Abbreviations: IAT, internet addiction tendency; L, left; MNI, Montreal Neurological Institute; R, right; TFCE, threshold-free cluster enhancement; WM, white matter

Table 4. Brain regions exhibiting significant positive correlations with IAT score

\begin{tabular}{|c|c|c|c|c|c|c|c|}
\hline \multirow[t]{2}{*}{ Anatomical area } & \multicolumn{3}{|c|}{$\begin{array}{l}\text { MNI } \\
\text { coordinates }\end{array}$} & \multirow[t]{2}{*}{$\begin{array}{l}\text { TFCE } \\
\text { value }\end{array}$} & \multirow{2}{*}{$\begin{array}{l}\text { Corrected } \\
\text { p value } \\
\text { (FWE) }\end{array}$} & \multirow{2}{*}{$\begin{array}{l}\text { Cluster } \\
\text { size } \\
\left(\mathrm{mm}^{3}\right)\end{array}$} & \multirow{2}{*}{$\begin{array}{l}\text { Activated areas, } \\
\text { deactivated areas during } \\
\text { the } 2 \text {-back task* }\end{array}$} \\
\hline & $x$ & $Y$ & Z & & & & \\
\hline $\begin{array}{l}\text { Left medial frontal } \\
\text { gyrus }\end{array}$ & -9 & 54 & -3 & 737.44 & 0.014 & 23112 & $0 \%, 97.5 \%$ \\
\hline $\begin{array}{l}\text { Left superior frontal } \\
\text { gyrus, medial part }\end{array}$ & -9 & 54 & 6 & 728.89 & 0.015 & & \\
\hline $\begin{array}{l}\text { Right anterior } \\
\text { cingulate }\end{array}$ & 6 & 39 & 6 & 675.55 & 0.019 & & \\
\hline
\end{tabular}

Abbreviations: IAT, internet addiction tendency; L, left; MNI, Montreal Neurological Institute; R, right; TFCE, threshold-free cluster enhancement; WM, white matter 
*Percentage of voxels showing significant activation or deactivation $(p<0.05$, false discovery rate (FDR) corrected at the voxel level) during the 2-back task among the 63 subjects sampled, from which the template of the diffusion image was created ${ }^{82}$.

\section{Figures}

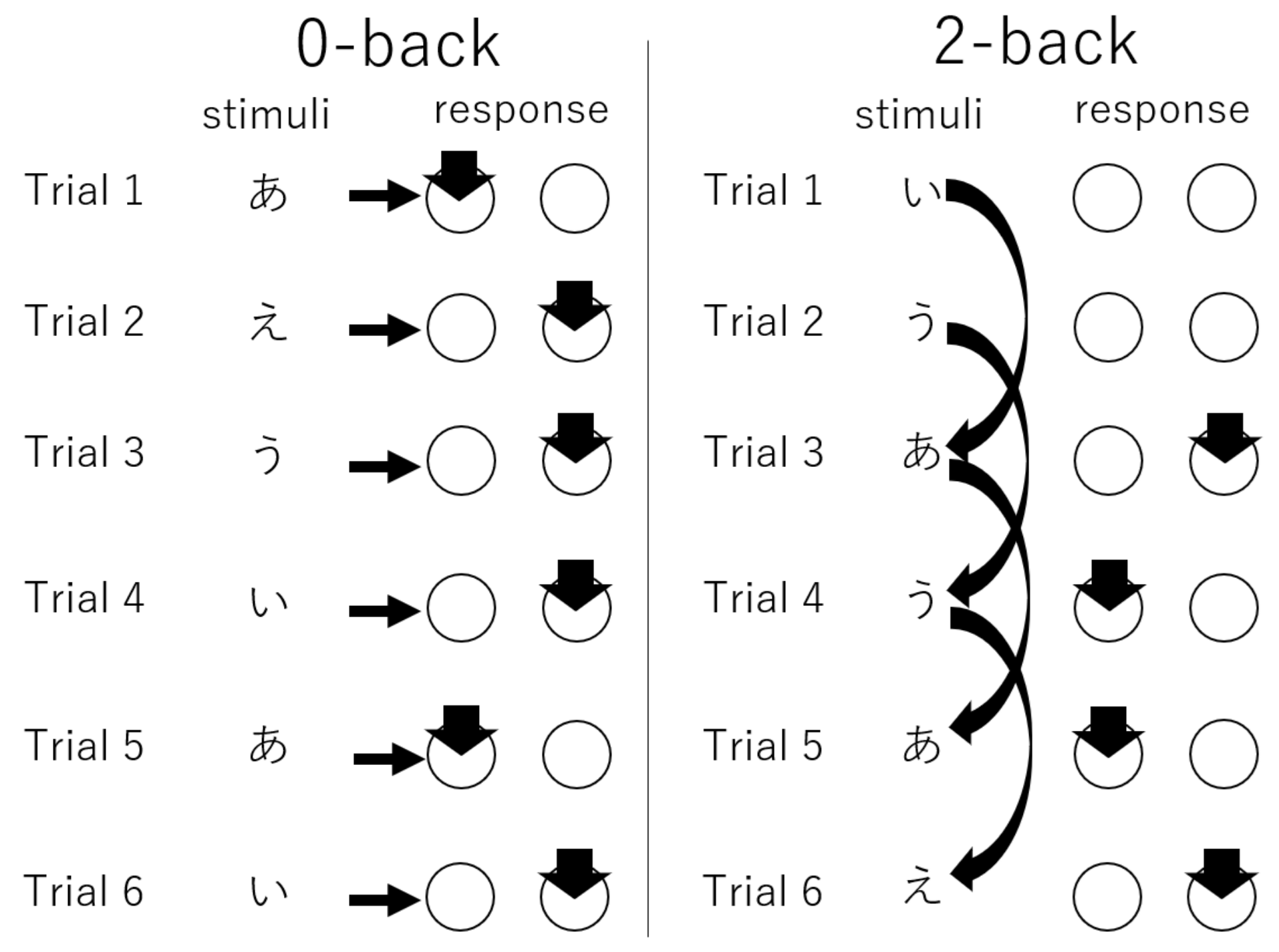

Figure 1

A schematic diagram of the procedures used for the N-back task. 


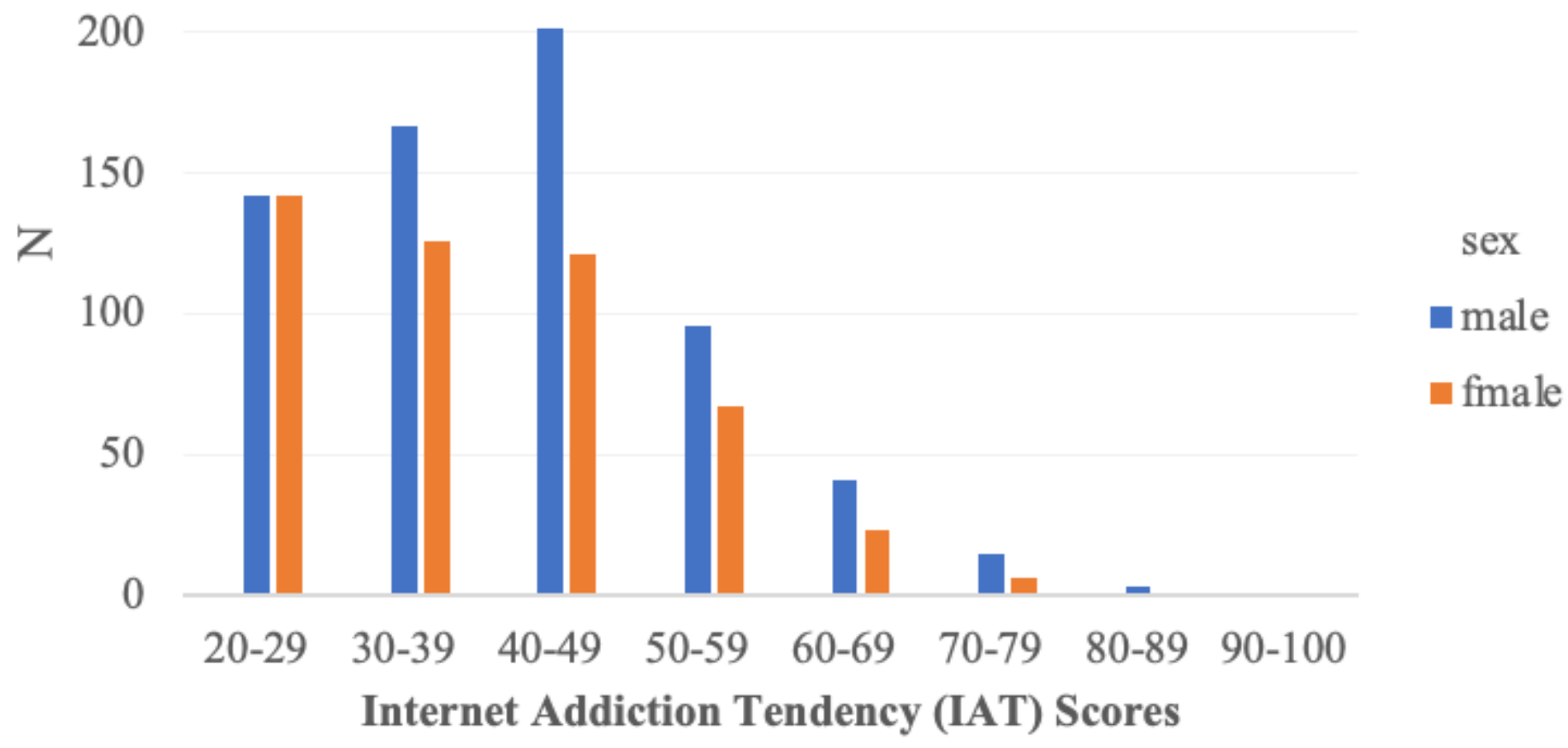

Figure 2

Distribution of internet addiction tendency (IAT) scores

a
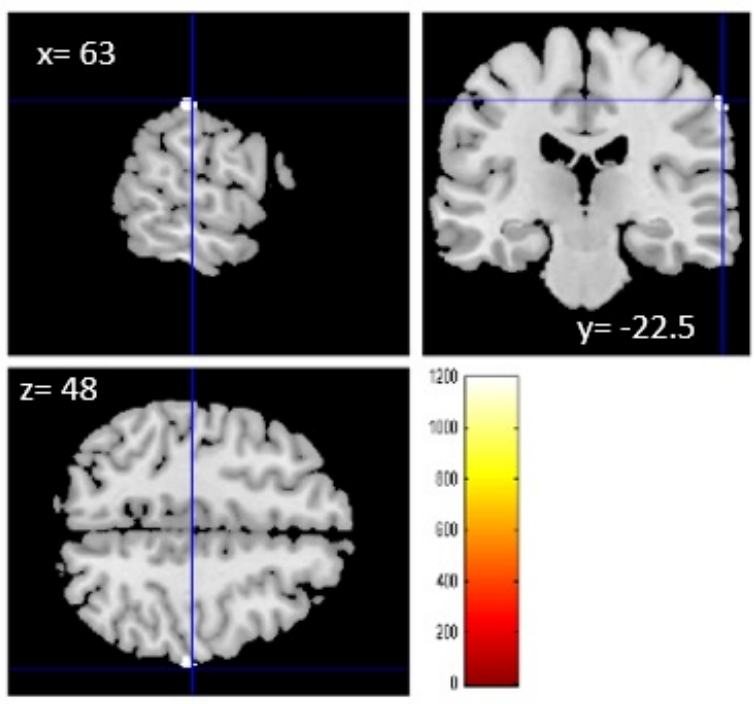

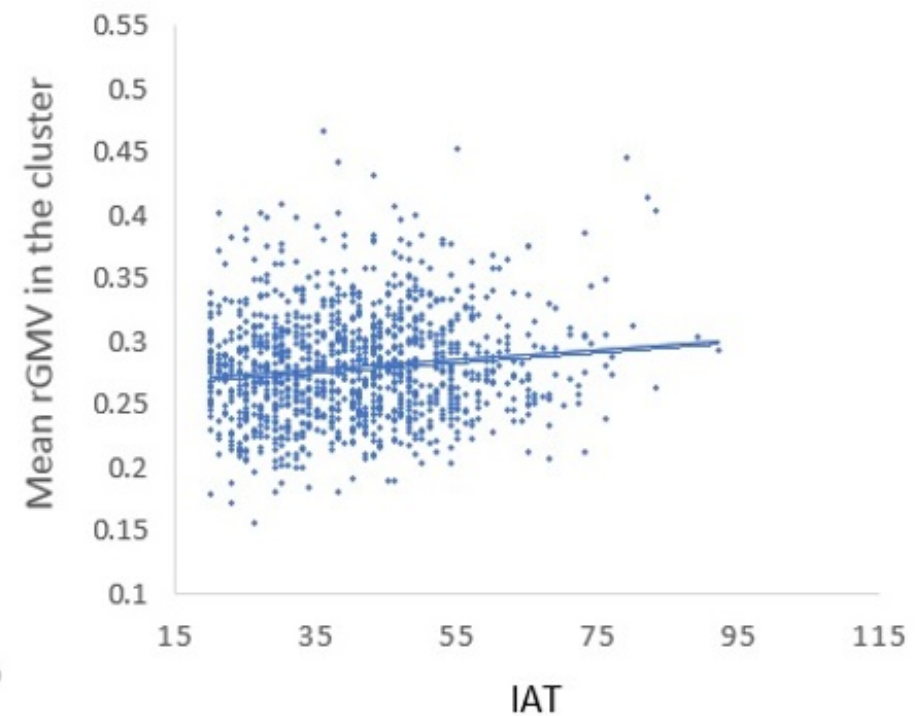

Figure 3

Regional gray matter volumes correlated with internet addiction tendency (IAT) score in young adults. (a) The panels show the areas of significant positive correlation between IAT score and rGMV. The results shown were obtained using a threshold of threshold-free cluster enhancement (TFCE) of $p<0.05$ based 
on 5,000 permutations. A significant positive correlation was found in the right supramarginal gyrus. (b) Scatterplot of the association between IAT score and mean rGMV values of the significant cluster. IAT is positively correlated with mean rGMV of the significant cluster in males $(r=0.10, p=0.01)$, and in females $(r=0.099, p=0.029)$.
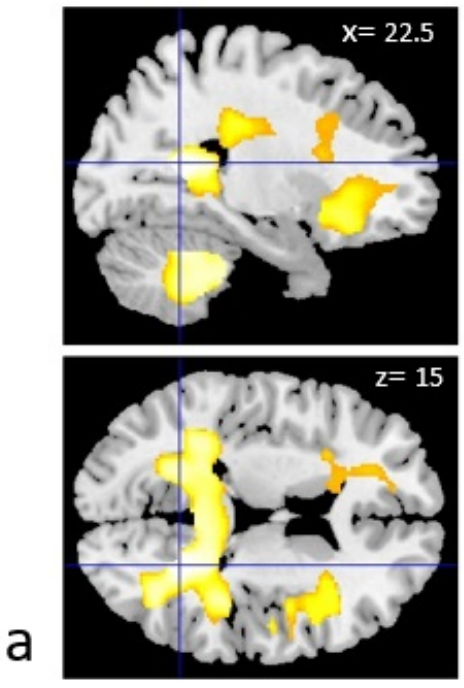
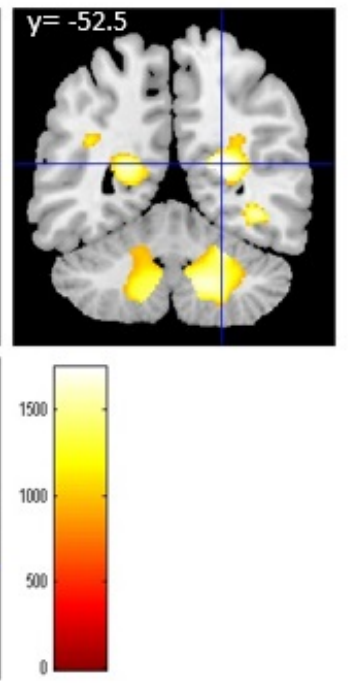

0.75

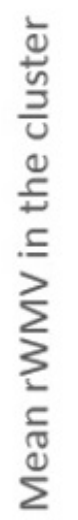

b

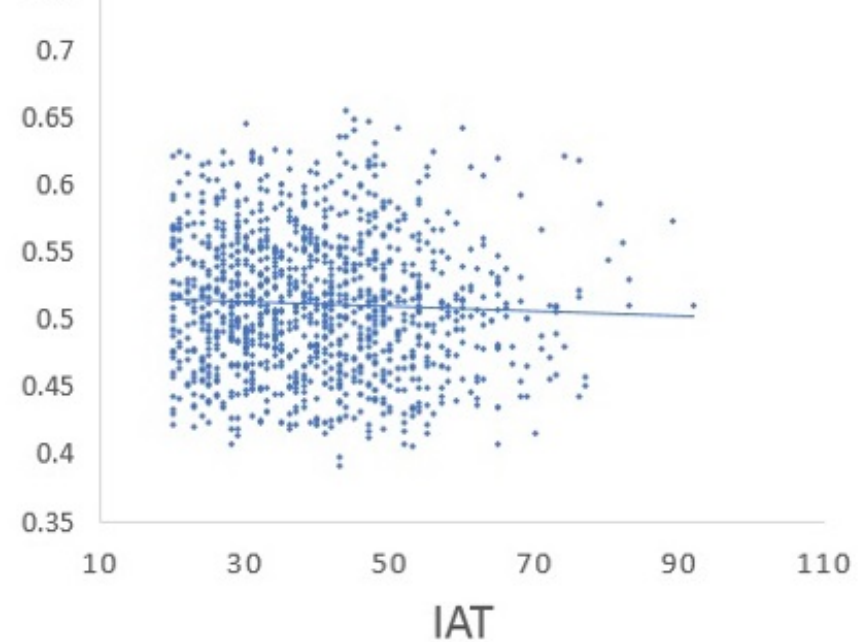

\section{Figure 4}

Regional white matter volumes correlated with internet addiction tendency (IAT) score in young adults. (a) The panels show the areas of significant negative correlation between IAT score and rWMV. The results shown were obtained using a threshold of threshold-free cluster enhancement (TFCE) of $p<0.05$ based on 5,000 permutations. Significant correlations were found in the sub-gyral area of the temporal lobe, superior temporal gyrus, extra-nuclear, lentiform nucleus, right cerebellum anterior lobe, cerebellar tonsil, right inferior frontal gyrus, sub-gyral of frontal lobe, and pons. (b) Scatterplot of the association between IAT score and mean rWMV values of the largest cluster. The simple correlation coefficient between mean rWMV signal of the significant cluster and IAT score is -0.045 . The association may look weak, but the partial correlation coefficient of this association when age, sex, and total intracranial volume were accounted for is -0.108 . IAT is negatively correlated with the mean rWMV of the significant cluster $1(r=$ $-0.113, p=0.003)$, significant cluster $2(r=-0.108, p=0.005)$, and significant cluster $3(r=-0.119, p=$ $0.002)$ in males. In addition, IAT has a slight negative correlation with the mean rWMV in cluster $1(-0.104$, $p=0.021)$ in females. 

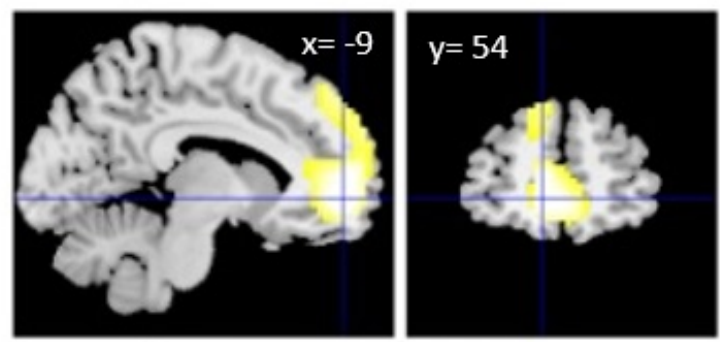

a
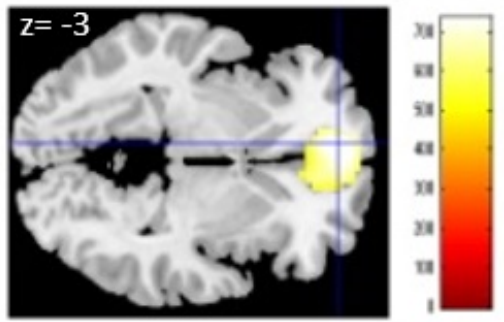

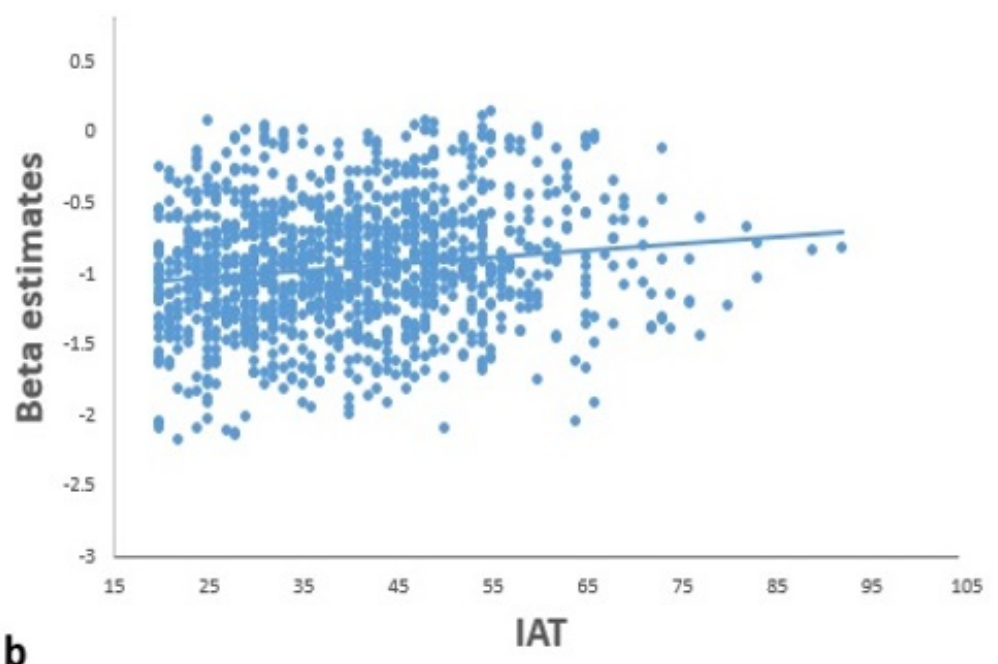

b

\section{Figure 5}

(a) Regional brain activity correlates with internet addiction tendency (IAT) scores. Regions with significant correlations between brain activity and IAT scores are overlaid on a "single subject" T1 image from SPM8. Results were obtained using a threshold of threshold-free cluster enhancement (TFCE) of $p<$ 0.05 based on 5,000 permutations. IAT scores were significantly and positively correlated with brain activity during the 2-back task in the default-mode network (medial frontal gyrus and anterior cingulum). (b) Scatterplot of the relationship between the IAT scores and brain activity during the 2-back task in the default-mode network. IAT showing a positive correlation with regional brain activity in males $(r=0.113, p$ $=0.003)$ and in females $(r=0.177, p=0.001)$.

$\mathbf{a}$
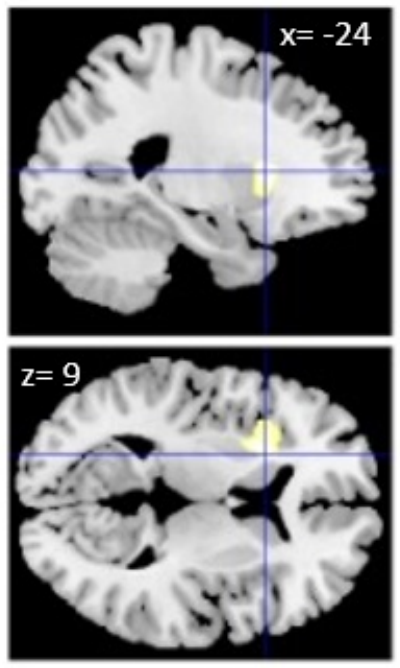
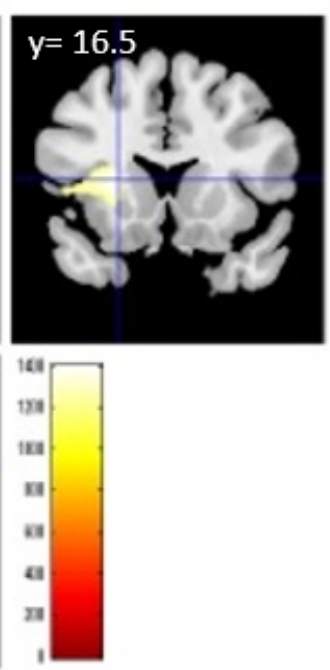

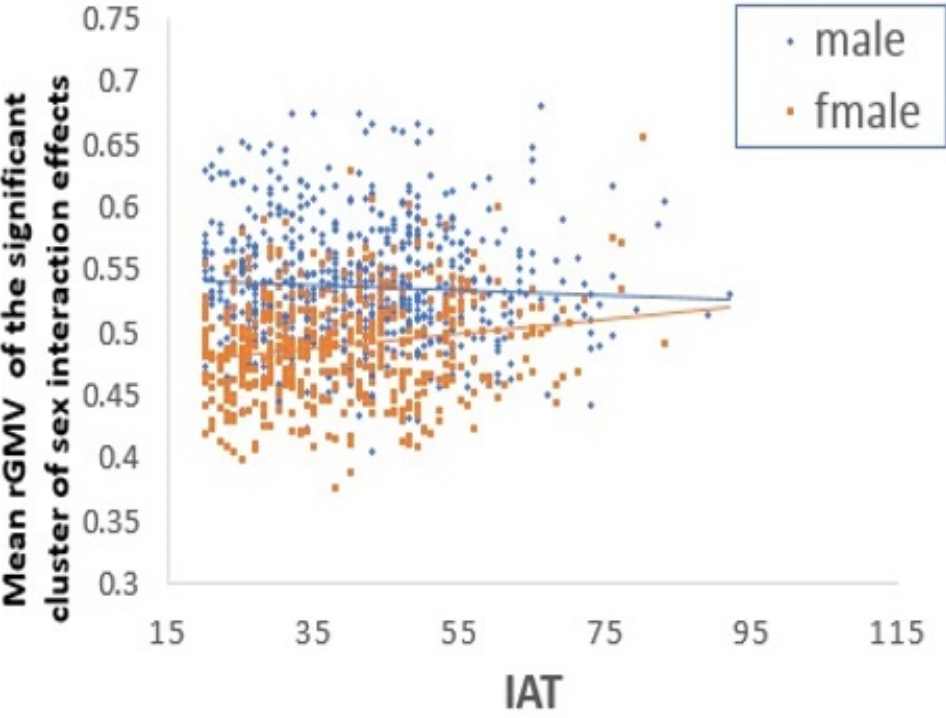

b IAT

Figure 6

Interaction between sex and internet addiction tendency (IAT) scores. (a) Whole-brain analyses of rGMV show significant effects of interaction between sex and the score of Young's IAT scale. Results were 
obtained using a threshold of threshold-free cluster enhancement (TFCE) of $p<0.05$ based on 5,000 permutations. The significant effects of interaction were found in the area around the left anterior insula and left lentiform. This interaction is positively correlated in females $(r=0.171, p=0.001)$, and not correlated in males $(r=-0.051, p=0.189)$. (b) Scatterplot of the mean rGMV of the significant cluster of sex interaction effects in the left basal ganglia and left anterior insula. 